\title{
MicroRNA-138 Suppresses Osteoblastic Differentiation of Valvular Interstitial Cells in Degenerative Calcific Aortic Valve Disease
}

\author{
Ping Lu, ${ }^{1} \mathrm{MD}$, Beibei Yin, ${ }^{2} \mathrm{MD}$ and Luqi Liu, ${ }^{1} \mathrm{MD}$
}

\begin{abstract}
Summary
The aim of this study was to explore the function of miR-138 in the pathogenesis of degenerative calcific aortic valve disease (DCAVD).

Aortic valve calcification tissue and normal tissue from DCAVD patients were collected to detect the expression of miR-138 by qRT-PCR, and immunohistochemical staining was performed to identify the phenotype of valve interstitial cells. QRT-PCR was performed to analyze the expression of miR-138, Runx2, MSX2, and ALP at day 7 after osteogenic differentiation. Alkaline phosphatase activity assay was performed at day 14 after osteogenic differentiation. Alizarin red staining was used to analyze the calcium nodule formation. TargetScan was used to predict potential targets of miR-138. QRT-PCR and Western blotting were performed to analyze the expression of FOXC1 in valve interstitial cells (VICs). The aortic valve calcification was evaluated by quantitative analysis of the velocity in the aortic annulus and transvalvular pressure gradients.

In this study, we demonstrated the role of miR-138 in VIC osteogenesis. QRT-PCR results revealed miR138 was significantly down-regulated in calcified aortic valves compared with non-calcified valves. MiR-138 overexpression inhibited VIC osteogenic differentiation in vitro, while down-regulation of miR-138 enhanced the process. Target prediction analysis and dual-luciferase reporter assay confirmed FOXC1 was a direct target of miR-138. Further research found FOXC1 overexpression promoted VIC osteogenic differentiation. In addition, animal experiments validated indirectly miR-138 could suppress aortic valve calcification.

Our findings suggest miR-138 could function as a new inhibitor of VIC osteogenic differentiation, which may act by targeting FOXC1.
\end{abstract}

Key words: FOXC1

(Int Heart J 2019; 60: 136-144)

$\mathrm{D}$ egenerative calcific aortic valve disease (DCAVD) is a valvular disease characterized by large amounts of calcium deposits in the valve in the elderly population. ${ }^{1)}$ DCAVD has a prevalence rate of $20 \%$ to $30 \%$ in people over age 65 , and up to $48 \%$ in those over age $85 .{ }^{2)}$ Changes in the form and structure of the heart valve connective tissue leads the valve and its affiliated normal function to be damaged. The main manifestations in the aortic valve are thickening and calcification, which eventually lead to cardiovascular diseases and related complications over time, such as left ventricular hypertrophy, congestive heart failure, angina, malignant arrhythmia, and sudden death. At present, the treatment of DCAVD relies mainly on surgical repair and replacement since there is no effective drug to prevent and cure the disease, which imposes an enormous economic burden on the patient and society. Therefore, it is meaningful and valuable for society to investigate the pathogenesis of DCAVD and to seek appropriate treatment of the disease. ${ }^{3)}$

DCAVD has traditionally been considered a passive and nonmodifiable wear-and-tear disease process that develops with advancing age. However, accumulating cellular and molecular biology studies have shown that the calcification process is active, involving a series of pathophysiological changes, such as chronic inflammatory reaction, lipid infiltration, calcium salt deposition, ${ }^{4)}$ neovascularization, and oxidative stress. ${ }^{5)}$ The pathogenesis of DCAVD remains unclear, and further study is needed.

Valve interstitial cells (VICs) are the main cells that regulate aortic valve structure and function in cardiac valve leaflets, ${ }^{6}$ which play a key role in calcific aortic valve disease progression." Studies have shown that the regulatory pathways of calcific aortic valve disease include the activation of VICs in addition to increased expression of related transcription factors that regulate the earliest events of valvulogenesis. ${ }^{8)}$ Venardos, et al. used isolated human VICs from aortic, pulmonary mitral, and tricuspid valves to determine the differences in TLR-4induced expression of an osteogenic phenotype, and demonstrated that TLR-4 stimulation induced an osteogenic

From the ${ }^{1}$ Department of Cardiac Surgery, Qianfoshan Hospital, Shandong University, Jinan, China and ${ }^{2}$ Department of Oncology, Qianfoshan Hospital, Shandong University, Jinan, China.

Address for correspondence: Luqi Liu, MD, Department of Cardiac Surgery, Qianfoshan Hospital, Shandong University, No. 16766 Jingshi Road, Jinan 250014, Shandong, China. E-mail: 1q131_liu@126.com

Received for publication February 5, 2018. Revised and accepted May 30, 2018.

Released in advance online on J-STAGE November 20, 2018.

doi: 10.1536/ihj.18-086

All rights reserved by the International Heart Journal Association. 
phenotype only in aortic VICs. ${ }^{9)}$ Other studies have found that VICs acquire an osteogenic phenotype during valve remodeling in calcific aortic valve disease and might be responsible for the formation of calcific nodules observed in the diseased valves. ${ }^{10)}$ The above study indicated that VICs differentiating into osteoblasts may provide an important basis for the development of DCAVD.

MicroRNAs are important short non-coding regulatory molecules in cells, which can participate in regulating many physiological and pathological processes. It has been reported that miR-204 suppressed the transformation of bone marrow mesenchymal cells into osteoblasts by inhibiting the expression activity of Runx2. ${ }^{11)}$ MiR-145 can regulate the process of osteodifferentiation by targeting transcription factor $\mathrm{Cbfb}^{12)} \mathrm{A}$ recent study of retrograde calcification valves found that miR-141 was involved in the transformation of the phenotype of the valvular cells through a BMP-dependent mechanism. ${ }^{13)}$ MiR-205 can inhibit the osteogenesis differentiation of vascular smooth muscle cells via regulation of Runx2 and Smad1 protein. ${ }^{14)}$ However, our knowledge concerning the function of miRNAs in DCAVD is still in its infancy, and the role of more miRNAs in osteoblast differentiation needs to be further studied.

MicroRNA 138 (miR-138) has been shown to regulate a number of biological processes, including developmental events tied to cell differentiation. ${ }^{15)}$ A study found that Runx 2 phosphorylation were decreased by the role of miR-138 with protein tyrosine kinase 2, which inhibited osteoblast differentiation eventually. ${ }^{16)}$ At present, the expression and function of miR-138 in cardiac valvular disease are still under investigation. Therefore, our findings about the exploration and verification of relevant microRNA may provide valuable information for the prevention and control of DCAVD at the molecular level.

\section{Methods}

Ethics statements: The study protocol was approved by the Ethical Committee of Qianfoshan Hospital of Shandong University and informed consent was obtained from the human donors. All experiments were performed in accordance with the relevant guidelines and regulations. All animal experimental procedures were approved by the institutional review board of Qianfoshan Hospital.

Calcific aortic valve collection: Samples were obtained from 10 DCAVD patients who had undergone aortic valve replacement. Exclusion criteria included non-stenotic, congenital aortic valve disease, autoimmune disease, genetic disease, and rheumatic aortic valve disease. The informed consent form was signed before the surgery. Two samples were taken from each patient: one sample was from a calcified valve, and the other was obtained from noncalcified tissue that served as a control. All samples were resected during the operation and immediately placed in pairs in liquid nitrogen for subsequent study. At the same time, pathological examinations of tissue samples from 10 patients were performed to confirm the accuracy of the tissue sampling and trimming.

MiRNA real-time quantitative PCR: MiR-138 was extracted using an miRVana extraction kit (Ambion, Austin,
TX). For miR-138 quantification, $10 \mathrm{ng}$ total RNA was reverse transcribed and amplified using an miRNA reverse transcription and detection kit (Applied Biosystems, USA). All results were normalized to U6 levels, which were determined using an ABI miRNA U6 assay kit (Applied Biosystems, USA).

VIC isolation and cell culture: Normal aortic valves were derived from patients who had undergone acute Stanford A aortic dissection. The non-leaflet tissues were carefully eliminated after effective removal of the endothelial layer of the aortic and ventricular aspects, and then the valves were immersed in $0.25 \%$ trypsin at $37^{\circ} \mathrm{C}$ for 5 minutes. The tissues were then cut into pieces and digested for an additional 2 hours at $37^{\circ} \mathrm{C}$. Primary VICs were obtained and seeded in growth medium (Dulbecco's modified eagle medium supplemented with penicillin and streptomycin, minimum essential medium (MEM) nonessential amino acid, sodium pyruvate, and 10\% FBS) at $37^{\circ} \mathrm{C}$ under a $5 \%$ carbon dioxide atmosphere. The purity of the VICs was confirmed by microscopic examination and evaluation of the expression of marker proteins.

Cell phenotype identification: Cell phenotype identification was performed in a primary valvular mesenchymal cell culture to the third generation. The VICs were first washed 2-3 times with PBS, fixed with more than $4 \%$ polyformaldehyde, rinsed 3 times with PBS, treated with $0.2 \%$ Triton- $100 \mathrm{X}$ at room temperature for 15 minutes, rinsed twice with PBS for 5 minutes each time, then cells were blocked with 1\% BSA and incubated overnight at $4{ }^{\circ} \mathrm{C}$ with primary antibodies at 1:100 dilution (rabbit anti$\alpha$-SMA and mouse anti-vimentin, Sigma). After being washed three times with PBS the secondary antibodies were added at 1:100 dilution (goat-anti-rabbit, Alexa Fluor 488 and goat-anti-mouse IgG, AlexaFlour 568 Sigma). After the cells were washed three times with PBS, images were acquired by confocal laser scanning microscopy (LSM510 META, Carl Zeiss, Heidenheim, Germany).

Transient transfection and cell treatments: VICs were seeded at a density of $3 \times 106$ cells in 6-well plates (Corning Costar, USA). When the cells reached $70-80 \%$ confluence, they were individually transfected with 50$\mathrm{nmol} / \mathrm{L} \mathrm{miR}-138 \mathrm{mimic}, 50-\mathrm{nmol} / \mathrm{L} \mathrm{miR}-138$ inhibitor, 50-nmol/L FOXC1 overexpression, or 50-nmol/L FOXC1 siRNA (GenePharma Co., Ltd, Shanghai, China) in OPTIMEMI reduced serum medium (Invitrogen, USA) using lipofectamine 2000 (Invitrogen, USA) according to the manufacturer's protocol. Transfection efficiency was measured in a preliminary test. Osteogenic differentiation was subsequently induced for 7 or 14 days after transfection by culturing cells in osteogenic differentiation medium (growth medium supplemented with 500-ng/mL BMP-2, 100-nmol/L dexamethasone, $50-\mu \mathrm{g} / \mathrm{mL}$ ascorbic acid, and 10-mmol/L $\beta$-glycerophosphate).

mRNA quantitative real-time PCR: The mRNA expressions of Runx2, MSX2, ALP, and FOXC1 were detected using qRT-PCR. Total RNA was extracted with TRIzol reagent (Invitrogen, USA). A Power SYBR Green RT-PCR Kit (Invitrogen, USA) and Bio-RAD CFX96 Real-Time System (Bio-RAD) were used for quantitative RT-PCR analysis. Data were normalized to the reference gene GAPDH for each cDNA sample. 
A

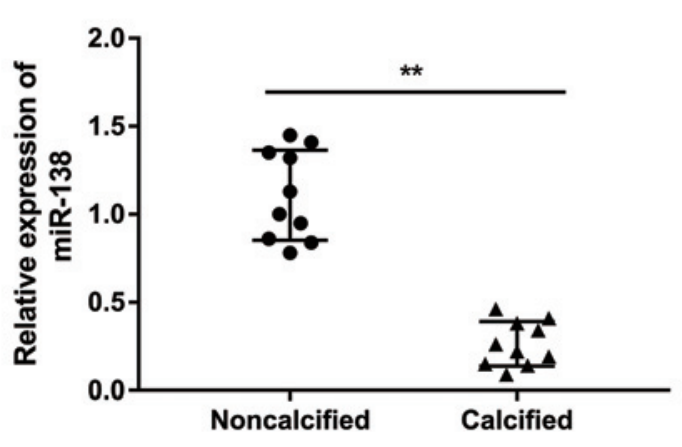

C

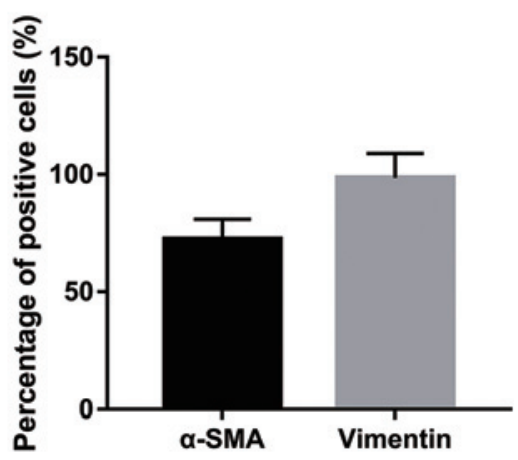

B

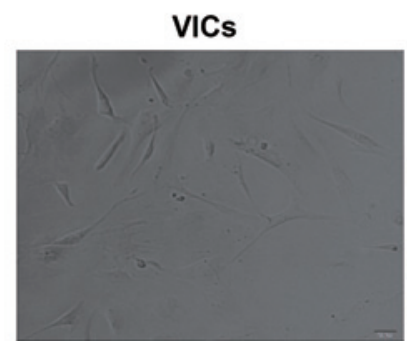

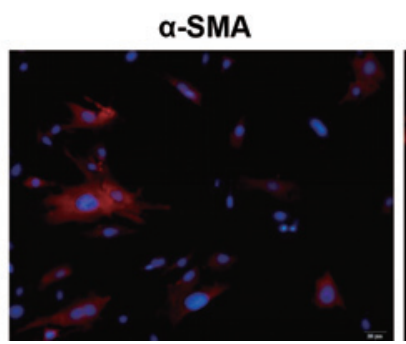

Figure 1. Characteristics and phenotypes of VICs. A: The expression of mir-138. B: The morphology of VICs. (Left: optical microscope; Middle: immunohistochemical staining of $\alpha$-SMA; Right: immunohistochemical staining of Vimentin) C: Quantification of positive staining of $\alpha$-SMA and Vimentin.

Alkaline phosphatase activity assay: The osteogenic phenotype was determined based on the alkaline phosphatase (ALP) activity, which is an early osteoblastic differentiation marker. The ALP activity assay was conducted after 7 days of osteogenic differentiation. Cells were washed twice with phosphate buffered saline solution (PBS) and lysed with $150 \mu \mathrm{L}$ of NP-40 lysis buffer (Beyotime, China). The cell lysates were quantified with a alkaline phosphatase assay kit (Beyotime, China) using pnitrophenyl phosphate (pNPP) as the substrate. In the presence of magnesium ions, pNPP was hydrolyzed by phosphatases to phosphate and p-nitrophenol. The rate of p-nitrophenol liberation is proportional to the ALP activity and can be measured photometrically. The ALP activity was measured using a spectrophotometer at $405 \mathrm{~nm}$.

Alizarin red staining: Alizarin red staining was conducted after 14 days of osteogenic differentiationto test matrix mineralization deposition, which appears at later stages of bone formation. In short, treated cells were washed twice with PBS, fixed in $95 \%$ ethanol for 10 minutes, washed with distilled water, and stained using Alizarin Red solution (1 g Tris and 0.1 g Alizarin Red (SigmaAldrich) in $100 \mathrm{~mL}$ ultrapure water; the $\mathrm{pH}$ was adjusted to 8.3 with $\mathrm{HCl}$ ) at $37^{\circ} \mathrm{C}$ for 30 minutes. Matrix calcification in alizarin red staining was manifested with red deposition.

Dual luciferase reporter assay: The 3'-UTR of human gene FOXC1 was amplified from human cDNA. The wide-type fragment containing the predicted miR-138 binding site and its mutant fragment, designed to carry sites for SacI (5' end) and XbaI ( 3 ' end) at their ends, were obtained from 3'-UTR of FOXC1. Amplicons were cleaved with SacI and $\mathrm{XbaI}$ and inserted between SacI and XbaI cleavage sites of pmirGLO vector (Promega, USA). A total of $293 \mathrm{~T}$ cells were selected on the basis of low endogenous miRNA expression. Cells were seeded in 24 -well plates. When $70 \%$ to $80 \%$ confluence was reached, $800 \mathrm{ng}$ of wild-type or mutant reporter and 20umol/L miR-138 mimic, inhibitor (GenePharma Co., Ltd, China) were co-transfected into the $293 \mathrm{~T}$ cells using Lipofectamine 2000 (Invitrogen, USA). Twenty-four hours after transfection, firefly and renilla luciferase activities were measured in cell lysates using a dual-luciferase reporter system.

Western blotting: The protein expressions of FOXC1 were measured using Western blotting. The transfected VIC samples were fixed in $4 \%$ paraformaldehyde for 30 minutes, and then blocked with $0.2 \%$ Triton X-100 and $3 \%$ goat serum in PBS. Cell lysate was separated by $12 \%$ sodium dodecyl sulfate-polyacrylamide gel electrophoresis (SDS-PAGE). Primary antibodies that included antiFOXC1 (ZSGB-BIO, China) and anti-GAPDH (ZSGB$\mathrm{BIO}$, China) were incubated overnight at $4^{\circ} \mathrm{C}$. After washing, the membranes were incubated with secondary antirabbit or anti-mouse horseradish peroxidase-conjugated antibodies (ZSGB-BIO, China) for 2 hours at room temperature.

Animal experiments: Agomir-138 and negative controls were purchased from GenePharma (GenePharma Co., Ltd, Shanghai, China). Twenty-eight 10-week-old male Balb/c mice were randomly divided into 4 groups. Each group contained 7 mice. Aortic valve calcification was induced by vitamin D3. The mice were given subcutaneous injections of vitamin D3 at a dose of $500,000-\mathrm{IU} / \mathrm{kg}$ body 
A

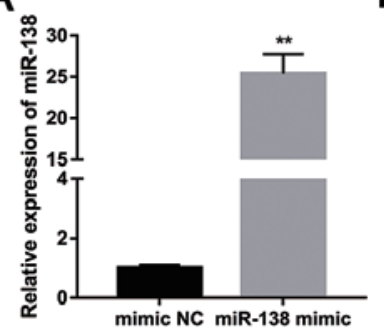

C

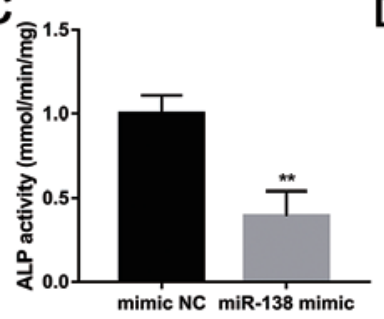

$\mathbf{F}$

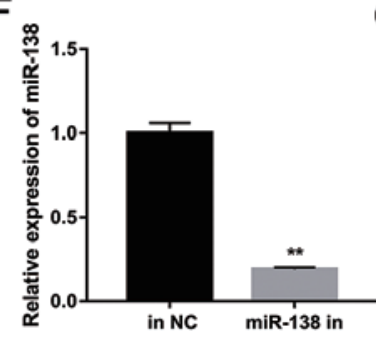

$\mathrm{H}$

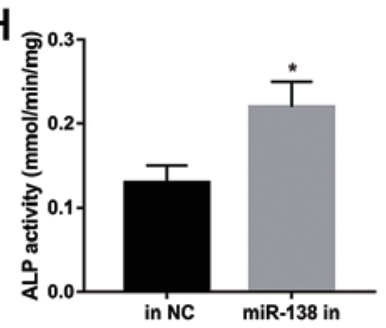

B

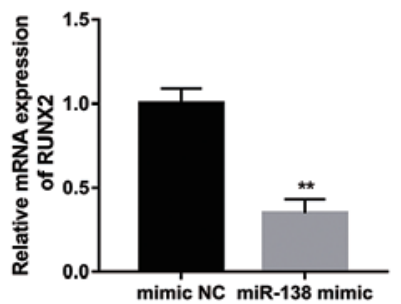

D

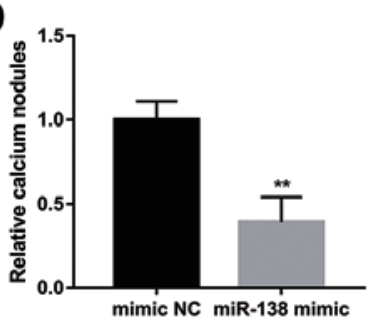

G

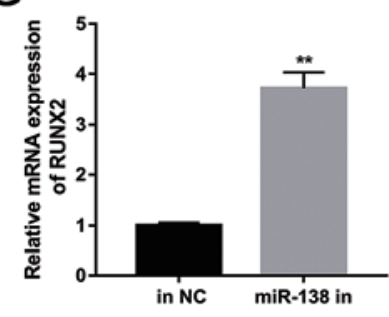

I

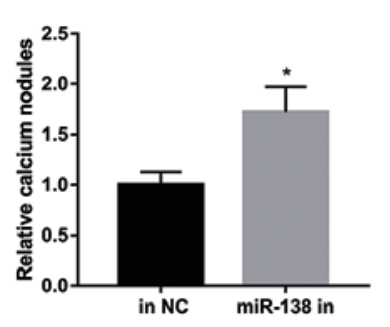

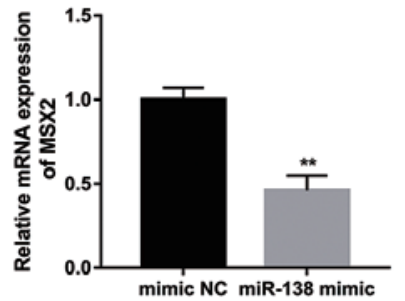

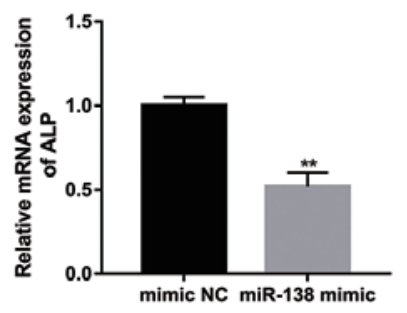

E
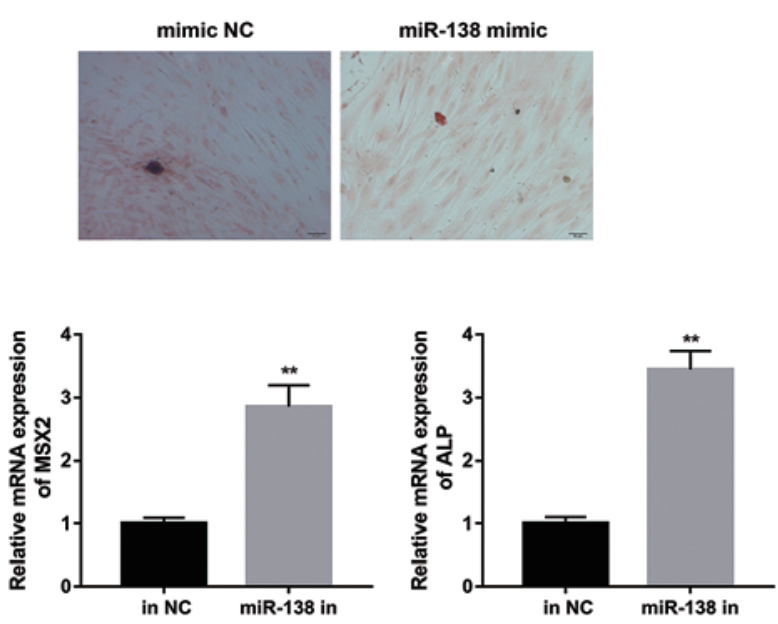

J

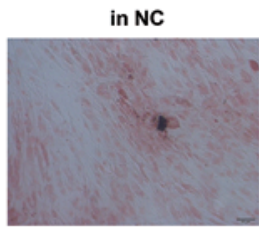

miR-138 in

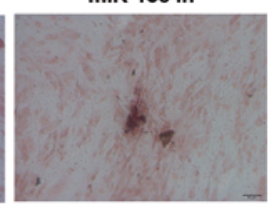

Figure 2. Overexpression of miR-138 inhibits VIC osteogenic differentiation while lowexpression of miR-138-5p promotes the process. A: qRT-PCR analysis of miR-138-5p expression in VICs transfected with miR-138 mimic at day 7. B: qRT-PCR analysis of Runx2, MSX2, and ALP expression at day 7 after osteogenic differentiation. C, D: ALP activity at day 14 after osteogenic differentiation. E: Alizarin red staining at day 14 after osteogenic differentiation. F: qRT-PCR analysis of miR-138-5p expression in VICs transfected with miR-138 inhibitor at day 7. G: qRT-PCR analysis of Runx2, MSX2, and ALP expression at day 7 after osteogenic differentiation. H, I: ALP activity at day 14 after osteogenic differentiation. J: Alizarin red staining at day 14 after osteogenic differentiation.

weight to induce aortic valve calcification and received 5$\mathrm{mg} / \mathrm{kg}$ body weight of agomir-138 and negative control through tail intravenous injection at days 1-3. Saline was used as a control. Six weeks later, velocity in the aortic annulus and transvalvular gradients were measured to indirectly evaluate aortic stenosis by using small animal echocardiography.

Statistical analysis: Each experiment was repeated in triplicate at least 3 times. All data were analyzed with SPSS 16.0 and are presented as the mean \pm standard deviation (SD). Student's t-test was used to analyze differences between two groups. One-way ANOVA analysis was used to perform multi-sample analysis. The nonparametric Mann-Whitney $U$ and Kruskal Wallis tests were performed when the sample size was smaller. Differences at $P<0.05$ were considered to be statistically significant.

\section{Results}

Characteristics and phenotypes of VICs: The aortic valve calcification and normal tissue from DCAVD patients were collected to detect the expression of miR-138 in retrograde calcified valves and normal smooth valve tissue. The results showed that miR-138 was significantly reduced in the retrograde calcified valve tissue $(P<0.01$, Figure $1 \mathrm{~A})$. The primary cells began to grow with adherence at about 12 hours of primary culture. Early cells were circular and complete, and then gradually spread out. Fusiform cells began to divide and proliferate, forming single layer VICs in a circinate arrangement after 5-7 days (Figure 1B). Cell phenotype identification was performed in the VICs from passage 3, and two marker proteins associated with VICs were characterized by immunohisto- 


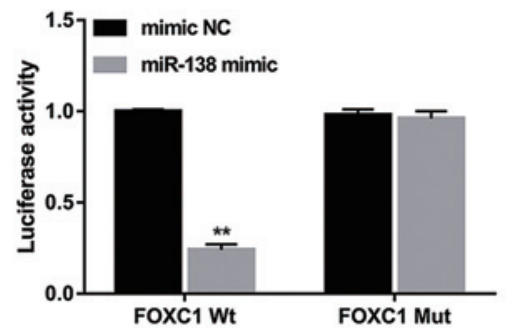

B

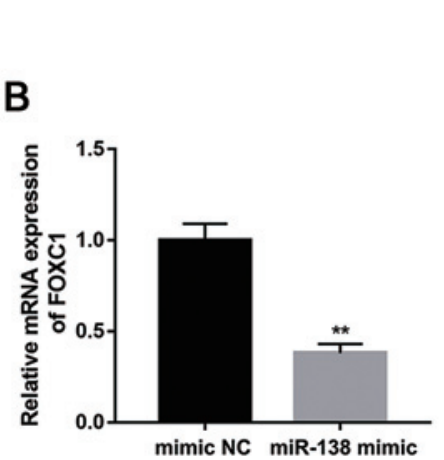

5'...UCCAAAAAUUCAGCUCACCAGCA.

3' gCcggacuaAguguUguggucGa
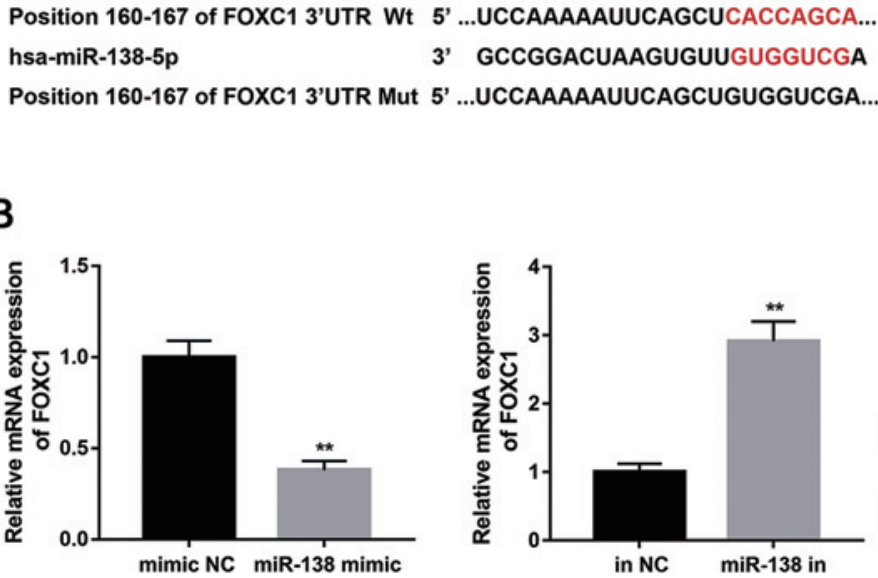

C

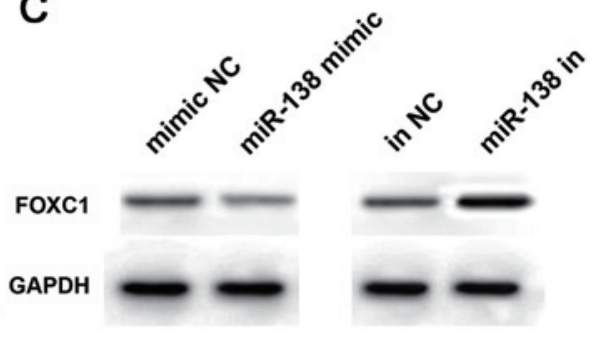

Figure 3. MiR-138 directly targets FOXC1. A: Schematic of the putative miR-138 target site in human FOXC1 3'-UTR; the 8 mutated nucleotides are colored red. B: qRT-PCR analysis of FOXC1 in VICs transfected with miR-138 mimic and inhibitor at day 7. C: Western blotting of FOXC1 in VICs transfected with miR-138 mimic and inhibitor at day 7.

chemical staining. VICs from passage 3 were positive for $\alpha$-SMA and vimentin (60\%-70\% and $100 \%$, respectively), as shown in Figure 1C.

miR-138 inhibits VIC osteogenic differentiation: First, we determined the efficiency of miR-138 transfection by qRT-PCR. The results showed that intracellular miR-138 levels were markedly up-regulated by an miR-138 mimic and significantly down-regulated by an miR-138 inhibitor (Figure 2A, F).

When VICs grew to about $70 \%$ to $80 \%$ of the culture plate area, a synthetic mimic and inhibitor of miR-138 were transfected into VICs, and calcification induction was performed. To further investigate whether miR-138 regulates VIC osteogenic differentiation, osteogenic capacity was examined by qRT-PCR, ALP activity, and alizarin red staining. The expressions of Runt-related transcription factor 2 (Runx2), msh homeobox-2 (MSX2), and alkaline phosphatase (ALP) were detected after transfection with an miR-138 mimic and inhibitor. Runx2 is a master differentiation marker of osteoblasts and regulates bone formation. ${ }^{17)}$ MSX2 is a homeodomain transcription factor relevant to bone development. ${ }^{18)}$ ALP is extensively used as a marker of osteoblast or osteogenic differentiation, which increases enzymatic activity to an osteoblastic phenotype. ${ }^{19)}$ The results showed that the miR-138 mimic significantly suppressed mRNA expression of Runx2, MSX2, and ALP while miR-138 inhibitor promoted the expression of Runx2, MSX2, and ALP after 7 days of calcification induction (Figure 2B, G). ALP activity was also detected and miR-138 overexpression significantly repressed ALP activity, while miR-138 inhibitor increased ALP activity after 14 days of osteogenic differentiation 14 days (Figure 2C, H). In addition, alizarin red staining was used to analyze calcium nodule formation. As shown in Figure $2 \mathrm{D}, \mathrm{E}, \mathrm{I}$, and $\mathrm{J}$ at the 14 th day of calcification induction, decreased calcium nodules were observed in the miR-138 overexpression group, while increased calcium nodules were observed in the miR-138 low-expression group. These data demonstrated that miR-138 plays a role in inhibiting VIC osteogenic differentiation.

miR-138 directly targets FOXC1: To clarify the molecular mechanism via which miR-138 regulates osteogenic differentiation of VICs, the miRNA target gene prediction site TargetScan was used to predict potential targets of miR-138. Among the candidates, we found a highly conservative and specific combination sequence between miR-138 and FOXC1 3'UTR (Figure 3A). Our results showed that the miR-138 mimic significantly repressed luciferase activity when cotransfected with reporter containing WT FOXC1 3'UTR but not MT FOXC1 3'UTR (Figure $3 \mathrm{~A}$ ). The synthetic mimic and inhibitor of miR-138 were transfected into VICs. The results showed that miR138 overexpression significantly suppressed mRNA expression of FOXC1, while the miR-138 inhibitor promoted the expression of FOXC1 (Figure 3B). As shown by Western blotting, FOXC1 protein expression was significantly decreased by the miR-138 mimic at day 7 after calcification induction (Figure 3C). The results of Western blotting were consistent with the quantitative PCR results. The above results indicated that miR-138 directly targets FOXC1.

FOXC1 promotes VIC osteogenic differentiation: To investigate the role of FOXC1 on osteogenic differentiation of VICs, overexpression vectors of FOXC1 were transfected into VICs and low-expression FOXC1 by transfecting VICs with SiRNA (Si-FOXC1). Our results showed that FOXC1 overexpression promoted osteogenic differentiation of VICs, indicated by mRNA expression of Runx2, MSX2, and ALP at day 7 after calcification induction (Figure 4A, B), and ALP activity (Figure 4C) and alizarin red staining (Figure 4D, E) at day 14 after calcification induction, while Si-FOXC1 suppressed osteogenic differentiation of VICs, as indicated by mRNA expression of Runx2, MSX2, and ALP (Figure 4F, G), ALP activity 

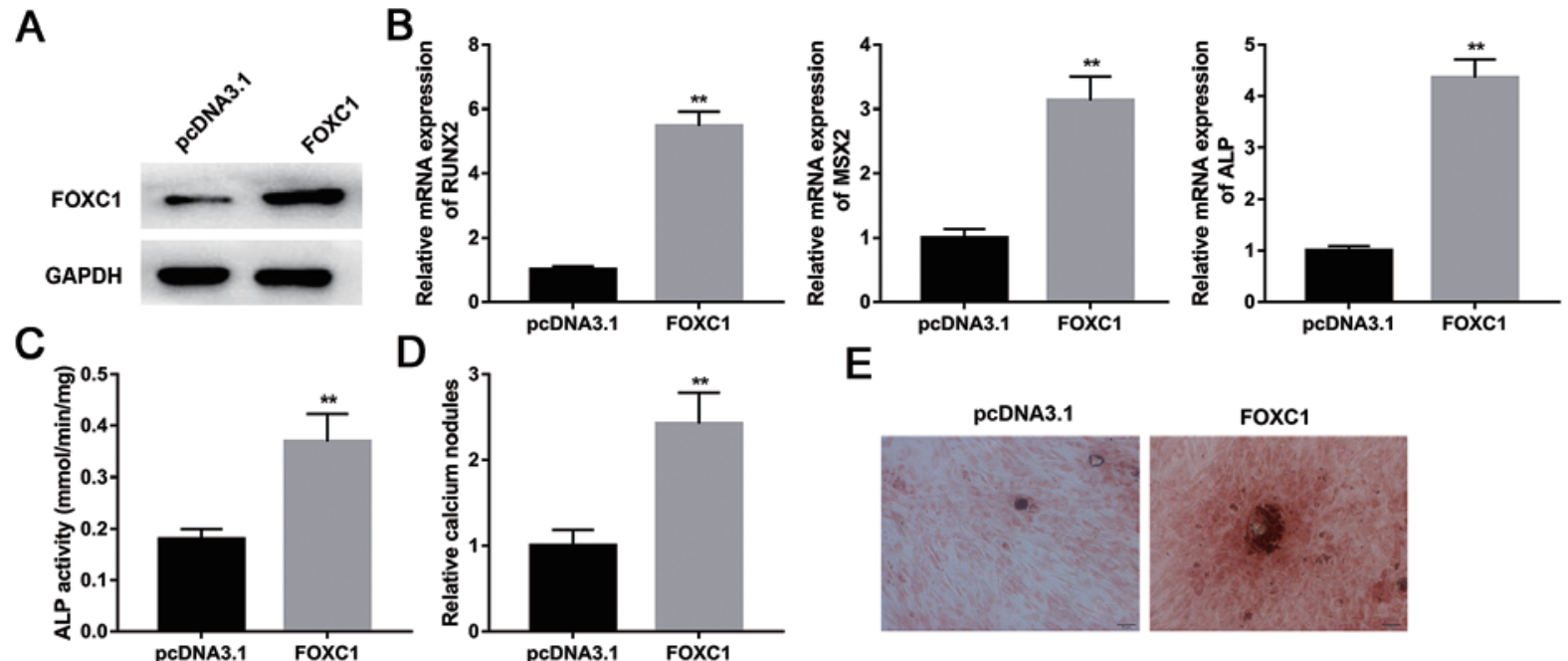

E
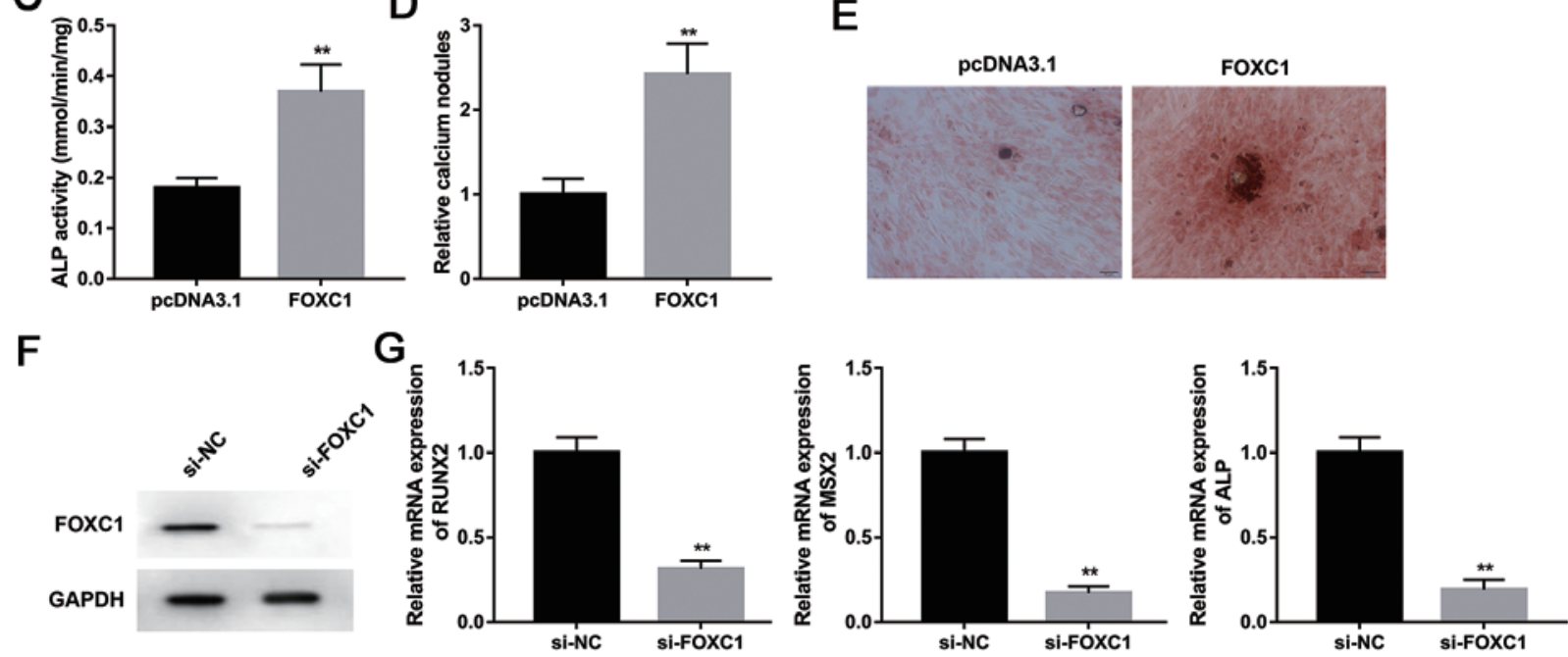

$\mathrm{H}$

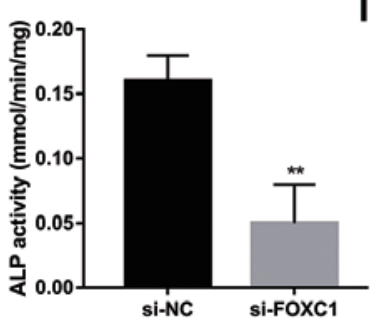

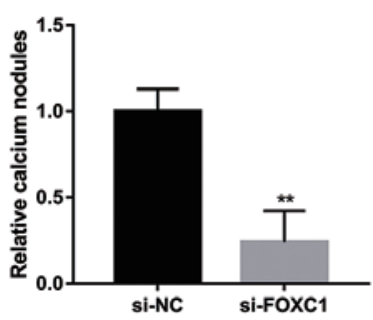

$\mathbf{J}$

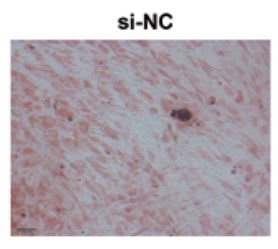

si-FOXC1

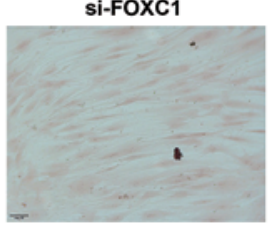

Figure 4. Overexpression of FOXC1 promotes VIC osteogenic differentiation while si-FOXC1 inhibits the process. A: Western blot analysis of FOXC1 in VICs transfected with overexpression of FOXC1 at day 7 after osteogenic differentiation. B: qRT-PCR analysis of Runx2, MSX2, and ALP expression at day 7 after osteogenic differentiation. C, D: ALP activity at day 14 after osteogenic differentiation. E: Alizarin red staining at day 14 after osteogenic differentiation. F: Western blot analysis of FOXC1 in VICs transfected with si-FOXC1 at day 7 after osteogenic differentiation. G: qRT-PCR analysis of Runx2, MSX2, and ALP expression at day 7 after osteogenic differentiation H, I: ALP activity at day 14 after osteogenic differentiation. J: Alizarin red staining at day 14 after osteogenic differentiation.

(Figure 4H), and alizarin red staining (Figure 4I, J). miR-138 negatively regulates FOXC1 during VIC osteogenic differentiation: To confirm that the effect of miR-138 during VIC osteogenic differentiation is mediated by targeting FOXC1, we transfected an miR-138 mimic into VICs after FOXC1 overexpression, and then proceeded with osteogenic differentiation. The results showed that miR-138 mimic-FOXC1 overexpression significantly inhibited mRNA expression of Runx2, MSX2, and ALP at day 7 after calcification induction (Figure 5A) and ALP activity at day 14 after calcification induction (Figure 5B). As shown in Figure 5C, D, decreased calcium nodules were observed in the miR-138 mimic-FOXC 1 overexpression group at day 14 after calcification induction. The results demonstrated that miR-138 regulates VIC osteogenic differentiation through FOXC1.
miR-138 inhibits aortic valve calcification in vivo: As shown in Figure $6 \mathrm{~A}$ and $\mathrm{B}$, vitamin $\mathrm{D}_{3}$-treated mice showed significantly higher velocity in the aortic annulus and higher transvalvular pressure gradients compared to sham mice, which indirectly confirmed aortic valve calcification was successfully induced. However, compared to the control group, miR-138 overexpression significantly reduced the velocity in the aortic annulus and the transvalvular pressure gradients. Compared to the control group, the results showed that miR-138 levels were markedly up-regulated in the vitamin D3+agomir-138 group (Figure 6C), and agomir-138 significantly suppressed the mRNA expression of FOXC1, Runx2, MSX2, and ALP in the vitamin D3-treated mice (Figure 6D-G). In conclusion, animal experiments demonstrated that miR-138 could alleviate aortic valve calcification in vivo. 

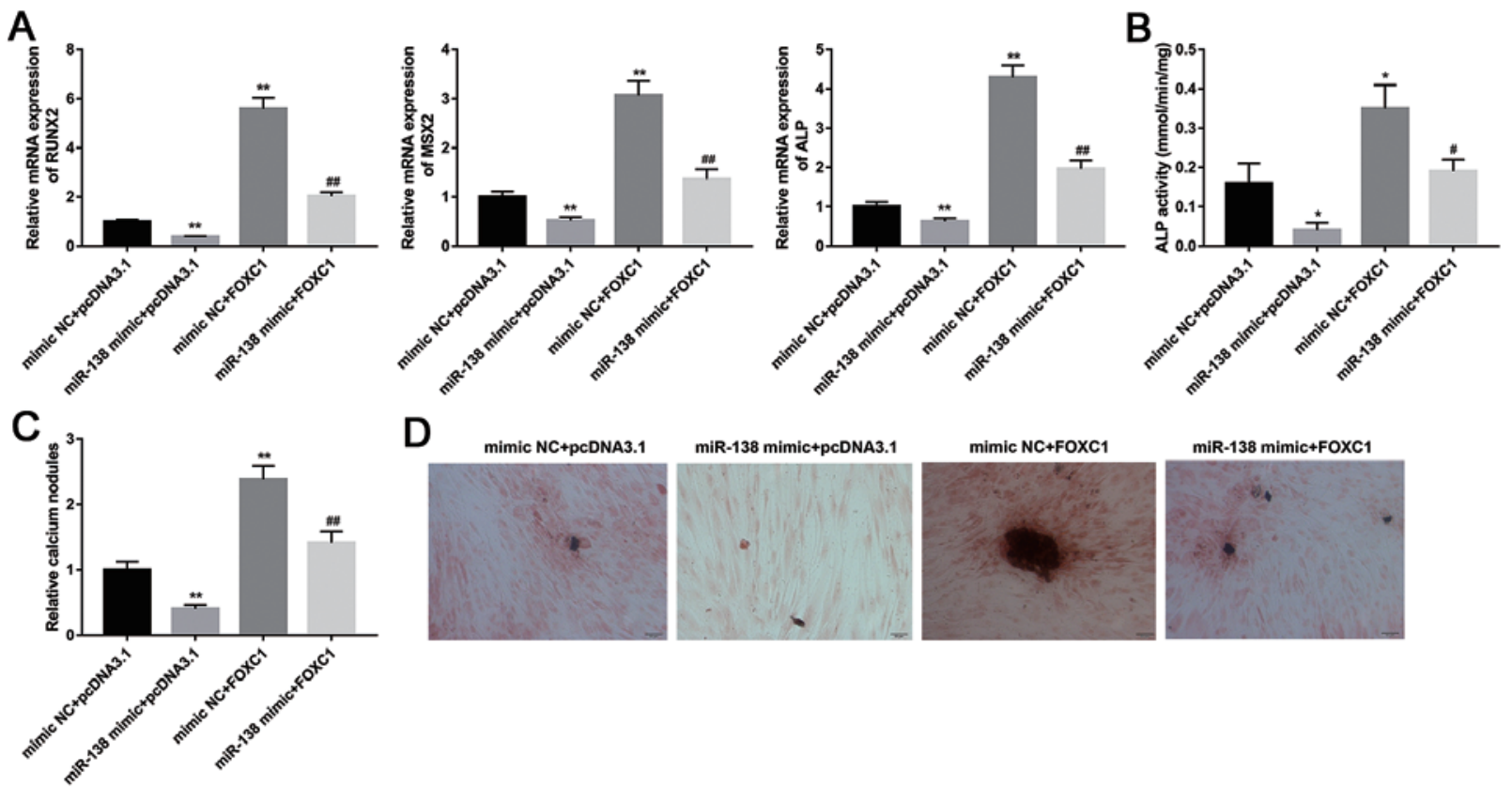

Figure 5. Regulation of FOXC1 by miR-138 during VIC osteogenic differentiation. A: qRT-PCR analysis of Runx2, MSX2, and ALP expression at day 7 after osteogenic differentiation. B: ALP activity at day 14 after osteogenic differentiation. C, D: Alizarin red staining at day 14 after osteogenic differentiation.

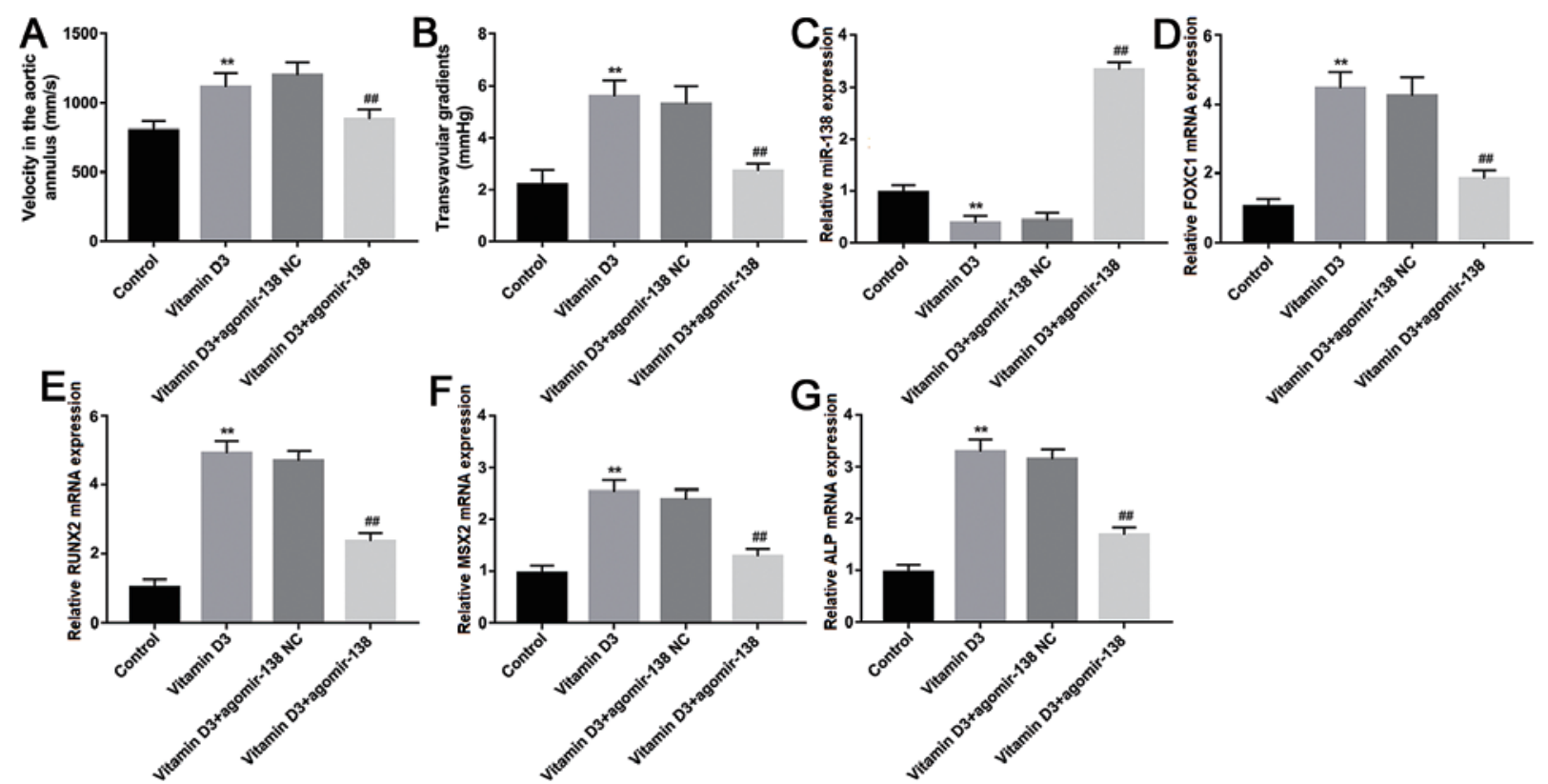

Figure 6. MiR-138 inhibits aortic valve calcification in vivo. A: Quantitative analysis of the velocity in the aortic annulus in each group. B: Quantitative analysis of transvalvular gradients in each group. C: QRT-PCR analysis of miR-138 expression in each group. D: QRT-PCR analysis of FOXC1 expression in each group. E: QRT-PCR analysis of Runx2 expression in each group. F: QRT-PCR analysis of MSX2 expression in each group. G: QRT-PCR analysis of ALP expression in each group.

\section{Discussion}

In this study, we identified the differentially expressed miRNAs of miR-138 by qRT-PCR in noncalcified and calcified aortic valves for the first time.
MiR-138 expression was significantly markedly downregulated in degenerative calcified aortic valves. To investigate the osteogenesis differentiation of miR-138 in aortic valve interstitial cells, we conducted osteogenesis induction after the overexpression and low expression of miR- 
138 in VICs, and we determined the effects of miR-138 on VIC osteodifferentiation at the cellular, protein, and mRNA levels. The results of ALP activity, alizarin red staining, and osteogene qRT-PCR demonstrated that miR138 inhibited VIC osteogenesis differentiation. Meanwhile, animal experiment results showed that the overexpression of miR-138 in vivo also plays an inhibitory role in the calcification of aortic valves, which further confirmed miR-138 suppressed the osteoblastic differentiation of valvular interstitial cells in vivo. To investigate the molecular mechanism of VIC osteodifferentiation, we predicted potential targets of miR-138 with TargetScan and found that FOXC1 contains a miR-138 binding site in its 3'UTR, while dual luciferase reporter assay indicated FOXC1 was a target gene of miR-138. Thus, our findings suggest that miR-138 plays a critical role in VIC osteogenic differentiation by directly targeting FOXC1.

MiRNAs are highly conserved evolutionarily in small single-stranded and non-coding RNA molecules, which act as key regulators of diverse biological processes by mediating translational repression or mRNA degradation of their target genes. ${ }^{20)}$ miRNAs have emerged as crucial regulators of diverse physiological and pathological processes, including cell proliferation, apoptosis, ${ }^{21)}$ inflammatory response, ${ }^{22)}$ and cancer, ${ }^{23)}$ and in particular cardiovascular diseases ${ }^{24}$. The role of miRNAs in DCAVD pathogenesis has been attracting more and more attention. MiR24 inhibited the osteogenesis differentiation of stem cells via the regulation of Tcf-1 protein expression, ${ }^{25)}$ while another report showed microRNA-449c-5p inhibits osteogenic differentiation of human VICs through a Smad4mediated pathway. ${ }^{26)}$ Huang, et al. reported that miRNA204/211 targets Runx2 in bone marrow-derived MSC, stimulates adipocyte differentiation, and inhibits osteoblastic differentiation. ${ }^{27)}$ Zhang, et al. confirmed that miR-30b can regulate the expression ofRunx2, Smadl, and Caspase3 , which play an important role in the calcification and apoptosis of human aortic valve interstitial cell calcification and apoptosis. ${ }^{28)}$ In this study, miR-138 was found and confirmed to negatively regulate the process of osteogenesis differentiation in human calcified aortic valve by targeting FOXC1.

It has been proven that miR-138 can play a crucial role in the regulation of multiple cell functions. Eskildsen, et al. found that miR-138 functions as a negative regulator of osteogenic differentiation of hMSCs, which results in suppression of the FAK-ERK1/2 signaling pathway. ${ }^{16)} \mathrm{Re}$ cently, miR-138 was reported as a negative regulator of adipocyte differentiation of human adipose tissue-derived MSCs. ${ }^{29)}$ Sun, et al. demonstrated that down-regulation of Noggin and miR-138 coordinately promoted osteogenesis of mesenchymal stem cells. ${ }^{30)}$ Another study showed that microRNA-138 directly targets TNFAIP8 and acts as a tumor suppressor in osteosarcomas. ${ }^{31)}$ In the present study, we identified miR-138 as a negative regulator of VIC osteoblast differentiation. Data obtained from in vitro experiments revealed that miR-138 overexpression inhibited osteoblast differentiation. These findings indicate that miR138 can target the FOXC1 gene, and regulate its expression level to regulate differentiation of human aortic interstitial cells, findings that are consistent with previous studies about the function of miR-138 in osteogenic differentiation.

Previous studies have shown that FOXC1 is closely related to osteogenesis differentiation. FOXC1 has a role in axial skeleton development by interacting with other transcription factors. ${ }^{32)}$ Inman, et al. proved the absence of FOXC1 affected the neural crest cell derived osteogenic pattern as osteoblasts develop ectopically in the maxillary prominence and fuse with the dentary bone. ${ }^{33)}$ The forkhead box transcription factor FOXC1 is required for normal development and patterning of bones originating from both endochondral and intramembranous origins. ${ }^{3)}$ Targeted deletion of the FOXC1 gene in mice results in numerous defects in the axial skeleton. ${ }^{34)}$ Furthermore, the expressions of two genes critical in the formation of the mouse craniofacial skeleton, Alx4 and Msx2, are reduced in FOXC1 mutant mice. ${ }^{35}$

\section{Conclusion}

In the present study, we have successfully demonstrated that FOXC1 protein plays a crucial part in regulating VIC osteogenic differentiation. At the same time, we also proved that FOXC1 is a direct target gene of miR138 using dual-luciferase reporter assay. Our study also confirmed the function of FOXC1 in regulating osteogenic differentiation of human aortic VICs.

\section{Disclosures}

Conflicts of interest: The authors declared no conflict of interest.

\section{References}

1. Otto CM. Calcific aortic stenosis--time to look more closely at the valve. N Engl J Med 2008; 359: 1395-8.

2. Otto CM, Lind BK, Kitzman DW, et al. Association of aorticvalve sclerosis with cardiovascular mortality and morbidity in the elderly. N Engl J Med 1999; 341: 142-7.

3. Rice R, Rice DPC, Olsen BR, et al. Progression of calvarial bone development requires Foxc1 regulation of Msx2 and Alx4. Dev Biol 2003; 262: 75-87.

4. Freeman RV, Otto CM. Spectrum of calcific aortic valve disease: pathogenesis, disease progression, and treatment strategies. Circulation 2005; 111: 3316-26.

5. Rajamannan NM, Evans FJ, Aikawa E, et al. Calcific aortic valve disease: not simply a degenerative process: A review and agenda for research from the National Heart and Lung and Blood Institute Aortic Stenosis Working Group. Executive summary: Calcific aortic valve disease-2011 update. Circulation 2011; 124: 1783-91.

6. Chester AH, Taylor PM. Molecular and functional characteristics of heart-valve interstitial cells. Philos Trans R Soc Lond B Biol Sci 2007; 362: 1437-43.

7. Liu AC, Joag VR, Gotlieb AI. The emerging role of valve interstitial cell phenotypes in regulating heart valve pathobiology. Am J Pathol 2007; 171: 1407-18.

8. Combs MD, Yutzey KE. Heart valve development: regulatory networks in development and disease. Circ Res 2009; 105: 40821.

9. Venardos N, Nadlonek NA, Zhan Q, et al. Aortic valve calcification is mediated by a differential response of aortic valve inter- 
stitial cells to inflammation. J Surg Res 2014; 190: 1-8.

10. Jian B, Narula N, Li QY, et al. Progression of aortic valve stenosis: TGF-beta1 is present in calcified aortic valve cusps and promotes aortic valve interstitial cell calcification via apoptosis. Ann Thorac Surg 2003; 75: 457-65.

11. Cui RR, Li SJ, Liu LJ, et al. MicroRNA-204 regulates vascular smooth muscle cell calcification in vitro and in vivo. Cardiovasc Res 2012; 96: 320-9.

12. Fukuda T, Ochi H, Sunamura S, et al. MicroRNA-145 regulates osteoblastic differentiation by targeting the transcription factor Cbfb. FEBS Lett 2015; 589: 3302-8.

13. Yanagawa B, Lovren F, Pan Y, et al. miRNA-141 is a novel regulator of BMP-2-mediated calcification in aortic stenosis. J Thorac Cardiovasc Surg 2012; 144: 256-62.

14. Qiao W, Chen L, Zhang M. MicroRNA-205 regulates the calcification and osteoblastic differentiation of vascular smooth muscle cells. Cell Physiol Biochem 2014; 33: 1945-53.

15. Jin Y, Chen D, Cabay RJ, et al. Role of microRNA-138 as a potential tumor suppressor in head and neck squamous cell carcinoma. Int Rev Cell Mol Biol 2013; 303: 357-85.

16. Eskildsen T, Taipaleenmaki H, Stenvang J, et al. MicroRNA-138 regulates osteogenic differentiation of human stromal (mesenchymal) stem cells in vivo. Proc Natl Acad Sci U S A 2011; 108: 6139-44.

17. Someya H, Fujiwara H, Nagata K, et al. Thymosin beta 4 is associated with RUNX2 expression through the Smad and Akt signaling pathways in mouse dental epithelial cells. Int $\mathrm{J}$ Mol Med 2015; 35: 1169-78.

18. Liu TM, Lee EH. Transcriptional regulatory cascades in Runx2dependent bone development. Tissue Eng Part B Rev 2013; 19: 254-63.

19. Mikami Y, Asano M, Honda MJ, Takagi M. Bone morphogenetic protein 2 and dexamethasone synergistically increase alkaline phosphatase levels through JAK/STAT signaling in C3H10T 1/2 cells. J Cell Physiol 2010; 223: 123-33.

20. Seok HY, Chen J, Kataoka M, et al. Loss of MicroRNA-155 protects the heart from pathological cardiac hypertrophy. Circ Res 2014; 114: 1585-95.

21. Yang X, Qin Y, Shao S, et al. MicroRNA-214 Inhibits left ventricular remodeling in an acute myocardial infarction rat model by suppressing cellular apoptosis via the phosphatase and tensin homolog (PTEN). Int Heart J 2016; 57: 247-50.

22. Lu X, Yin D, Zhou B, et al. MiR-135a Promotes inflammatory responses of vascular smooth muscle cells from $\mathrm{db} / \mathrm{db}$ mice via downregulation of FOXO1. Int Heart J 2018; 59: 170-9.

23. Brennecke J, Hipfner DR, Stark A, et al. bantam encodes a developmentally regulated microRNA that controls cell proliferation and regulates the proapoptotic gene hid in Drosophila. Cell 2003; 113: 25-36.

24. Wang D, Zhai G, Ji Y, et al. microRNA-10a Targets T-box 5 to Inhibit the Development of Cardiac Hypertrophy. Int Heart J 2017; 58: 100-6.

25. Zhao W, Wu C, Dong Y, et al. MicroRNA-24 Regulates osteogenic differentiation via targeting T-cell factor-1. Int J Mol Sci 2015; 16: 11699-712.

26. Xu R, Zhao M, Yang Y, et al. MicroRNA-449c-5p inhibits osteogenic differentiation of human VICs through Smad4mediated pathway. Sci Rep 2017; 7: 8740.

27. Huang J, Zhao L, Xing L, et al. MicroRNA-204 regulates Runx 2 protein expression and mesenchymal progenitor cell differentiation. Stem Cells 2010; 28: 357-64.

28. Zhang M, Liu X, Zhang X, et al. MicroRNA-30b is a multifunctional regulator of aortic valve interstitial cells. J Thorac Cardiovasc Surg 2014; 147: 1073-80 e2.

29. Yang Z, Bian C, Zhou H, et al. MicroRNA hsa-miR-138 inhibits adipogenic differentiation of human adipose tissue-derived mesenchymal stem cells through adenovirus EID-1. Stem Cells Dev 2011; 20: 259-67.

30. Sun XK, Zhou J, Zhang L, et al. Down-regulation of Noggin and miR-138 coordinately promote osteogenesis of mesenchymal stem cells. J Mol Histol 2017; 48: 427-36.

31. Zhou Z, Li Z, Shen Y, et al. MicroRNA-138 directly targets TNFAIP8 and acts as a tumor suppressor in osteosarcoma. Exp Ther Med 2017; 14: 3665-73.

32. Motojima M, Tanimoto S, Ohtsuka M, et al. Characterization of kidney and skeleton phenotypes of mice double heterozygous for Foxc1 and Foxc2. Cells Tissues Organs 2016; 201: 380-9.

33. Inman KE, Purcell P, Kume T, et al. Interaction between Foxc1 and Fgf8 during mammalian jaw patterning and in the pathogenesis of syngnathia. PLoS Genet 2013; 9: e1003949.

34. Kume T, Deng K-Y, Winfrey V, et al. The forkhead/winged helix gene Mf1 is disrupted in the pleiotropic mouse mutation congenital hydrocephalus. Cell 1998; 93: 985-96.

35. Mavrogiannis LA, Antonopoulou I, Baxova A, et al. Haploinsufficiency of the human homeobox gene ALX4 causes skull ossification defects. Nat Genet 2001; 27: 17-8. 